\title{
Immune checkpoint inhibitors in extensive disease small cell lung cancer
}

\begin{abstract}
Small cell lung cancer (SCLC) is a very aggressive disease. Until now, little progress has been made in systemic treatments. Recently presented early phase clinical trials with immune checkpoint inhibitors (blockade of the cytotoxic T-lymphocyte antigen-4 (CTLA4 ) and blockade of the programmed cell death-1 (PD-1) receptor) have shown promising results. In this review, evidence on immune checkpoint blockade for SCLC is presented.
\end{abstract}

Keywords: SCLC; immune checkpoint inhibitors, lung cancer
Volume 7 Issue 6 - 2017

\author{
Gamze Gokoz Dogu \\ Department of Internal Medicine, Division of Medical Oncology, \\ School of Medicine, University of Pamukkale, Turkey
}

Correspondence: Gamze Gokoz Dogu, Department of Internal Medicine, Division of Medical Oncology, Faculty of Medicine, University of Pamukkale, Denizli, Turkey, Email ggd2882@gmail.om

Received: February 21, 2017 | Published: April 20, 2017

\section{Introduction}

Lung cancer accounts for $12 \%$ of all new cases of cancers worldwide, it is the second most common cancer in men and women, and represents the most common cause of cancer-related deaths worldwide. ${ }^{1,2}$ Small cell lung cancer (SCLC) accounts for approximately $10-15 \%$ of all lung cancers. ${ }^{3}$ Prognosis in SCLC is poor. Median survival without treatment has been reported as 2-4 months. ${ }^{4}$ Until now treatment of SCLC remains a tremendous challenge for oncologists. The prognosis of small cell cancer has not changed much over the last few decades, with the median survival time ranging from 15to 20 months for LD-SCLC (limited disease) and 8 to 13 months for ED-SCLC (extensive disease). ${ }^{1,5}$ The activation of the immune system is recognised as an important treatment strategy against cancer, recently. ${ }^{6}$

Blockade of the immune inhibitory ligand PD-L1 by a monoclonal antibody produced both durable tumor regression and prolonged disease stabilization in patients with melanoma, head and neck, NSCLC, and bladder carcinomas, renal-cell cancer and ovarian cancer recently. ${ }^{7-10} \mathrm{~A}$ recent report showed that programmed death ligand 1 (PD-L1) is expressed in tumor-infiltrating immune cells in the SCLC stroma, indicating that these patients may potentially respond to PD-1 inhibitors. ${ }^{11}$ The results of PD-L1 inhibitors demonstrated this treatment was more beneficial than vaccines for SCLC in the last few years.

CTLA-4 has been the first immune checkpoint receptor to be targeted by a therapeutic agent. CTLA-4 is expressed on activated T cells whose function is to down-regulate T-cell activity. ${ }^{13}$ Ipilimumab (a fully human monoclonal IgG1) blocks the interaction between CTLA-4 and its ligands CD80 and CD86. Ipilimumab was studied in combination with carboplatin and paclitaxel as first-line treatment for extensive stage (ED) SCLC in a randomised phase II clinical trial. ${ }^{13}$ In this study, 334 patients were randomized 1: 1: 1 to receive either concurrent-ipilimumab regimen; phased-ipilimumab regimen or control regimen. Phased ipilimumab, concurrent ipilimumab and control, respectively, were associated with median median PFS of 5.2, 3.9 and 5.2 months; median OS of 12.9, 9.1 and 9.9 months. Overall rates of grade $3 / 4$ irAEs (immun-related advers events) were 17,21 and $9 \%$ for phased ipilimumab, concurrent ipilimumab and control, respectively. ${ }^{14} \mathrm{~A}$ phase 3 trial comparing the efficacy of ipilimumab plus etoposide/platinum versus etoposide/platinum in patients with newly diagnosed ED-SCLC (NCT01450761) is currently ongoing. Ipilimumab is currently being investigated in the STIMULI study as a consolidation treatment, in combination with nivolumab, after concurrent chemotherapy for patients with LDSCLC (NCT02046733).

PD-1 is the major immune checkpoint receptor that inhibits T-cell activation and regulates immunosuppression through binding of its ligand, PD-L1. Pembrolizumab is an anti-PD-1 monoclonal antibody that blocks the PD-1/PD-L1 pathway KEYNOTE-028 is an ongoing phase $\mathrm{Ib}$ multicohort basket study evaluating pembrolizumab in PD-L1-positive patients with advanced Solid tumors and which have not responded to current therapy or for which current therapy is not appropriate. A total of 24 patients with pretreated extensivestage disease (ED) small-cell lung cancer (SCLC) and tumor PD-L1 positivity (membranous PD-L1 expression in $\geq 1 \%$ of tumour and associated inflammatory cells or positive staining in stroma using the 22C3 antibody clone) were enrolled in KEYNOTE-028 and received pembrolizumab at the dose of $10 \mathrm{mg} / \mathrm{kg}$ intravenously once every 2 weeks for up to 2 years, until progression or unacceptable toxicity. Primary end points were overall response rate (ORR) and safety and tolerability. Among 24 evaluable patients, ORR was 33\% (95\% confidence interval [CI], 16\%-55\%), including 1 complete and 7 partial responses. Median duration of response was 19.4 months (range, 3.6$20+$ months). Median PFS was 1.9 months ( $95 \%$ CI, 2-6 months). The 6- and 12-month PFS rates were $29 \%$ and $24 \%$, respectively. Median OS was 9.7 months ( $95 \%$ CI, 4.1 months to not reached). The 6- and 12 -month OS rates were $66 \%$ and $38 \%$, respectively. The safety profile was consistent with previous experience of pembrolizumab in other tumour types. Sixteen of $24(67 \%)$ patients experienced treatment-related adverse events (AEs). The most common treatmentrelated AEs were arthralgia $15 \%$, asthenia $15 \%$, nausea $10 \%$ and rash $10 \%$. There was one case of grade 2 autoimmune thyroiditis resulting in treatment interruption. Two patients had grade $\geq 3$ AEs including one treatment-related death from colitis. They found that there was no significant difference relationship between level of PD$\mathrm{L} 1$ expression and frequency of response $(\mathrm{p}=0.235)$ [15]. A phase II trial by European Organization for research and Treatment of Cancer will evaluate platinum/etoposide \pm pembrolizumab (NCT02580994) in patients with ED-SCLC. Another phase II trial is currently ongoing and investigates maintenance pembrolizumab after four cycles of induction platinum/etoposide (NCT02359019). 
A phase I/II open-label study (CheckMate 032) of nivolumab with or without ipilimumab for treatment of recurrent SCLC was evaluated. In this study, an objective response was achieved in ten $(10 \%)$ of 98 patients receiving nivolumab $3 \mathrm{mg} / \mathrm{kg}, 14(23 \%)$ of 61 receiving nivolumab $1 \mathrm{mg} / \mathrm{kg}$ plus ipilimumab $3 \mathrm{mg} / \mathrm{kg}$, and ten (19\%) of 54 receiving nivolumab $3 \mathrm{mg} / \mathrm{kg}$ plus ipilimumab $1 \mathrm{mg} / \mathrm{kg}$. Grade 3 or 4 treatment-related adverse events occurred in $13(13 \%), 18(30 \%)$ $10(19 \%)$, respectively. the most commonly reported grade 3 or 4 treatment-related adverse events were increased lipase and diarrhoea Six $(6 \%)$ patients in the nivolumab $3 \mathrm{mg} / \mathrm{kg}$ group, seven (11\%) in the nivolumab $1 \mathrm{mg} / \mathrm{kg}$ plus ipilimumab $3 \mathrm{mg} / \mathrm{kg}$ group, and four (7\%) in the nivolumab $3 \mathrm{mg} / \mathrm{kg}$ plus ipilimumab $1 \mathrm{mg} / \mathrm{kg}$ group discontinued treatment due to treatment-related adverse events. Two patients who received nivolumab $1 \mathrm{mg} / \mathrm{kg}$ plus ipilimumab $3 \mathrm{mg} / \mathrm{kg}$ died from treatment-related adverse events (myasthenia gravis and worsening of renal failure), and one patient who received nivolumab $3 \mathrm{mg} / \mathrm{kg}$ plus ipilimumab $1 \mathrm{mg} / \mathrm{kg}$ died from treatment-related pneumonitis. There was no correlation between PD-L1 expression (1\% cut-off) and ORR. On the basis of these encouraging phase $1 / 2$ data, phase 3 studies CheckMate 451, NCT02538666 and CheckMate 331, NCT02481830 in SCLC are currently ongoing.

Other immune checkpoint inhibitors that are being studied for the treatment of SCLC include durvalumab (MEDI4736), a PD-L1 antibody and tremelimumab, a CTLA-4 antibody. These agents are being investigated in an open-label nonrandomised phase I safety study of patients with advanced solid malignancies including SCLC (NCT02537418).

\section{Conclusion}

Over the past four decades, clinical developments in the treatment of SCLC have had a relatively modest impact on overall survival Immune checkpoint inhibitors have shown promise in many tumor types, including SCLC. Preliminary data for pembrolizumab, nivolumab and ipilimumab show promising antitumour activity, with durable responses and manageable AE profiles. Further research is necessary to ascertain the long-term safety and efficacy of immune checkpoint inhibitors.

\section{Funding}

None.

\section{Acknowledgements}

None.

\section{Conflicts of interests}

The authors declare that there is no conflict of interest.

\section{References}

1. van Meerbeeck JP, Fennell DA, De Ruysscher DK. Small-cell lung cancer. Lancet. 2011;378(9804):1741-1755.

2. Siegel RL, Miller KD, Jemal A. Cancer statistics 2016. CA Cancer J Clin. 2016;66:7-30.

3. Van Meerbeeck JP, Fennell DA, De Ruysscher DK. Small cell lung cancer. Lancet. 2011;378(9804):1741-1755.

4. Kato Y, Ferguson TB, Bennett DE, et al. Oat cell carcinoma of the lung. A review of 138 cases. Cancer. 1969;23(3):517-524.

5. Lally BE, Urbanic JJ, Blackstock AW, et all Small cell lung cancer: have we made any progress over the last 25 years? Oncologist. 2007;12(9):1096-1104.

6. Swann JB, Smyth MJ. Immune surveillance of tumors. J Clin Invest. 2007;117:1137-1146.

7. Brahmer JR, Tykodi SS, Chow LQ, et al. Safety and activity of antiPD-L1 antibody in patients with advanced cancer. $N$ Engl $J$ Med. 2012;366(26):2455-2465.

8. Topalian SL, Hodi FS, Brahmer JR, et al. Safety, activity, and immune correlates of anti-PD-1 antibody in cancer. $N$ Engl $J$ Med. 2012;366(26):2443-2454.

9. Sznol M, Chen L. Antagonist antibodies to PD-1 and B7-H1 (PDL1) in the treatment of advanced human cancer. Clin Cancer Res. 2013;19(5):1021-1034.

10. Rizvi NA, Hellmann MD, Snyder A, et al. Cancer immunology. Mutational landscape determines sensitivity to PD-1 blockade in nonsmall cell lung cancer. Science. 2015;348(6230):124-128.

11. Ilie M, Hofman V, Long E, et al. PD-L1 expression in primary tumor and circulating tumor cells in patients with small cell lung carcinomas. Presented at the American Association of Cancer Research, New Orleans, LA, 2016;20 (abstr 2220).

12. Spigel DR, Socinski MA. Rationale for chemotherapy, immunotherapy, and checkpoint blockade in SCLC. J Thorac Oncol. 2013;8(5):587-598.

13. Salama AK, Hodi FS. Cytotoxic T-lymphocyte-associated antigen-4. Clin Cancer Res. 2011;17(14):4622-4628.

14. Reck M, Bondarenko I, Luft A, et al. Ipilimumab in combination with paclitaxel and carboplatin as first-line therapy in extensive-diseasesmall-cell lung cancer: results from a randomized, double-blind, multicenter phase 2 trial. Ann Oncol. 2013;24(1):75-83.

15. Ott PA, Fernandez ME, Hiret S, et al. Pembrolizumab (MK-3475) in patients (pts) with extensive-stage small cell lung cancer (SCLC): preliminary safety and efficacy results from KEYNOTE-028. $J$ Clin Oncol. 2015;33(suppl; abstr 7502). 